\title{
ASYMPTOTIC SOLUTION OF A SMALL PARAMETERED 2-D INTEGRAL EQUATION ARISING FROM A CONTACT PROBLEM OF ELASTICITY BASED ON THE SOLUTION OF A 2-D INTEGRAL EQUATION
}

\author{
TIAN QUAN YUN
}

(Communicated by J. Marshall Ash)

\begin{abstract}
Asymptotic solution of a 2-D integral equation of constant kernel with small parameter $\varepsilon$,

$$
\int_{0}^{\pi} \int_{-\infty}^{\infty} p d s d \psi+\varepsilon r \int_{0}^{\pi} \int_{-\infty}^{\infty} p d s \cos \psi d \psi=G(r)
$$

which occurs in a more exact form of Hertz's contact problem in elasticity, is presented in this paper based on the solution of a 2-D integral equation

$$
\int_{0}^{\pi} \int_{-\infty}^{\infty} p d s d \psi=F(r)
$$

with constant kernel, and the unknown function $p=p(s, \psi)=p(t, \phi)$ is subjected to the following two constraints:

$$
\begin{aligned}
p(t, \phi) & =p(t) \quad \forall \phi, \\
p(s, \psi)=0 \quad \text { for }(s, \psi) & =(t, \phi) \notin E=\{(t, \phi) \mid t \leq a\}
\end{aligned}
$$

where $(s, \psi)$ are local polar coordinates with origin at $M(r, 0)$, with $(r, 0)$ measured by global polar coordinates $(t, \phi)$ with origin at $O(0,0)$. A more exact solution of Hertz's contact problem is found as an example.
\end{abstract}

\section{INTRODUCTION}

Contact problems have important applications in mechanical and civil engineering. Hertz's contact problem is a well-known classical problem in elasticity, and it is introduced by many textbooks (e.g., [1]). Hertz (1882) found, by conjecture, the solution of a contact problem of pressure distribution $p$ between two elastic balls in contact, governed by the integral equation

$$
\int_{0}^{\pi} \int_{-\infty}^{\infty} p d s d \psi=\left(\alpha-\beta r^{2}\right) /\left(k_{1}+k_{2}\right),
$$

Received by the editors July 30, 1992 and, in revised form, July 27, 1993.

1991 Mathematics Subject Classification. Primary 45E99; Secondary 73C99, 45M05, 44A15.

Key words and phrases. Hertz's contact problem, Radon transform, Abel integral equation, asymptotic expansion.

This project was supported by the National Natural Science Foundation of China. 


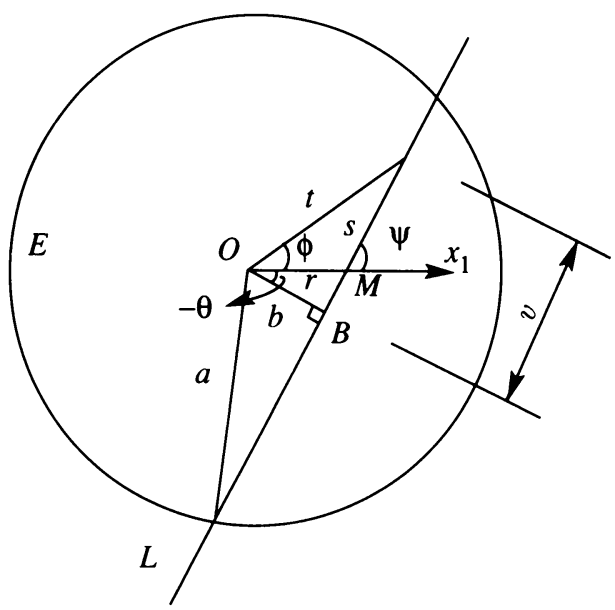

FIGURE 1

where $\alpha, \beta, k_{1}$, and $k_{2}$ are constants, and the unknown pressure $p$ is subjected to the constraints

$$
\begin{aligned}
p(t, \phi) & =p(t) \quad \forall \phi, \\
p(s, \psi)=0 \quad \text { for }(s, \psi) & =(t, \phi) \notin E=\{(t, \phi) \mid t \leq a\},
\end{aligned}
$$

where $(s, \psi)$ are local polar coordinates with origin at $M(r, 0)$ with $(r, 0)$ measured by global polar coordinates $(t, \phi)$ with origin at $O(0,0)$ (Figure 1). However, as pointed out in [2], the classical Hertz's integral equation (1) is not an exact equation for describing contact solids since the horizontal displacement is neglected there. An exact integral equation of Hertz's contact problem, where both vertical and horizontal components of displacement of points at contact surfaces due to pressure $p$ are taken into consideration, was derived in [2] and is shown as

$$
\begin{aligned}
& \iint p d s d \psi+\varepsilon r \iint p d s \cos \psi d \psi+\delta\left(\iint p d s \cos \psi d \psi\right)^{2} \\
& \quad=\left(\alpha-\beta r^{2}\right) /\left(k_{1}+k_{2}\right),
\end{aligned}
$$

where the limits of integration are the same as (1) and are omitted for convenience, and $\alpha, \beta, k_{1}, k_{2}, \varepsilon$, and $\delta$ are constants. To conserve space, the expressions of these constants are omitted and we just mention that $0<\delta \ll$ $\varepsilon \ll 1$. If the small high-order term $\delta$ is omitted and the right-hand side is replaced by a more general function $G(r)$, then (4) becomes

$$
\int_{0}^{\pi} \int_{-\infty}^{\infty} p d s d \psi+\varepsilon r \int_{0}^{\pi} \int_{-\infty}^{\infty} p d s \cos \psi d \psi=G(r) .
$$

If the small term $\varepsilon$ is further neglected, then (5) can be written as

$$
\int_{0}^{\pi} \int_{-\infty}^{\infty} p d s d \psi=F(r),
$$

where $F(r)$ is a given function. If we take $F(r)=\left(\alpha-\beta r^{2}\right) /\left(k_{1}+k_{2}\right)$, then (6) reduces to (1). 
Many studies of differential equations with small parameters can be found, but studies of integral equations with small parameters are rarely seen. This paper studies the solution of the 2-D integral equation (5) with small parameter $\varepsilon$ by asymptotic expansion based on the solution of the 2-D integral equation (6). Although Hertz found the solution of integral equation (1) by a conjecture, the solution of Hertz's integral equation (1) has not been derived in a strict mathematical way. In $\S 2$ the solution of the 2-D integral equation (6), which is more general than Hertz's integral equation (1), is derived by introducing the Radon transform, changing variables, and using the property of symmetry shown in (2). In $\S 3$ the integral equation (5) is solved by asymptotic expansion. Finally, as an example of the application of the asymptotic solution of (5), a comparison between the asymptotic solution and Hertz's solution is given, which shows that the classical Hertz solution is not exact enough for engineering applications but that the asymptotic solution is better in satisfying the criterion of the integral equation (5).

\section{Solution of integral equation (6) SUbJeCted to (2) AND (3)}

No published paper concerned with integral equation (6) subjected to constraints (2) and (3) has been found. Here, we try to solve this problem as follows.

CT (computerized tomography) is a revolutionary medical instrument. Its appearance shocked the medical world, winning for its inventors the 1979 Nobel Medicine Prize. The Radon transform could be considered as a mathematical fundamental of image reconstruction from projections, especially for CT. Therefore, the Radon transform is an interesting and fairly hot topic at the moment. Here, we introduce the Radon transform to our problem. A Radon transform of function $f$ of two variables $(s, \psi)$ in local polar coordinates is also a function of two variables $(b, \theta)$ but in global polar coordinates and is defined [3] by

$$
[R f](b, \theta) \equiv \int_{-\infty}^{\infty} f\left(\sqrt{b^{2}+v^{2}},-\theta+\tan ^{-1}(v / b)\right) d v .
$$

The Radon transform of $f$ represents a line integration of $f(s, \psi) \quad(=f(t, \phi))$ along a line $L$ with angle $\psi$ (Figure 1), where $b$ is the distance between $O(0,0)$ and $L, \theta$ is the angle between $O B$ and the $x_{1}$-axis, $O B \perp L$, and the function $f$ satisfies that

$$
f(s, \psi)=0 \quad \text { for }(s, \psi) \notin E,
$$

where $E$ is the domain of function $f$.

The relationship between local polar coordinates $(s, \psi)$ and global polar coordinates $(t, \phi)$ is

$$
\begin{aligned}
& s \cdot \sin \psi=t \cdot \sin \phi, \\
& s \cdot \cos \psi=t \cdot \cos \phi-r .
\end{aligned}
$$

Now, according to the definition of the Radon transform of a function, the 
left-hand side (L.H.S) of (6) can be written as

$$
\begin{aligned}
\text { L.H.S. } & =\int_{0}^{\pi} \int_{-\infty}^{\infty} p(s, \psi) d s d \psi=\int_{0}^{\pi} \int_{-\infty}^{\infty} p(t, \phi) d s d \psi \\
& =\int_{0}^{\pi} \int_{-\infty}^{\infty} p\left(\sqrt{v^{2}+b^{2}},(-\theta)+\tan ^{-1}(v / b)\right) d s d \psi \\
& =\int_{0}^{\pi}[R p](b, \theta) d \psi=\int_{-\pi / 2}^{\pi / 2}[R p](b, \theta) d \theta \\
& =[R p]\left(b_{0}, \theta_{0}\right)=\int_{-V_{0}}^{V_{0}} p\left(\sqrt{v^{2}+b^{2}}\right) d v,
\end{aligned}
$$

where $t^{2}=b^{2}+v^{2}, s=v+r \cdot \sin (-\theta), \psi+(-\theta)=\pi / 2, V_{0}^{2}=a^{2}-b_{0}^{2}$, $b_{0}=r \cdot \cos \left(-\theta_{0}\right), \theta_{0} \in[-\pi / 2, \pi / 2]$, and (2), (3), and the theorem of integral mean value of functions have been used. Both $b_{0}$ and $\theta_{0}$ can be determined from the right-hand side of (6) for a given $F(r)$.

Let

$$
w^{2}=b_{0}^{2}+v^{2}
$$

and, transforming the variable $v$ to $w, d v=(w / v) d w$; using (2), we have

$$
\text { L.H.S. }=2 \pi \int_{b_{0}}^{a} w\left(w^{2}-b_{0}^{2}\right)^{-1 / 2} p(w) d w .
$$

In order to obtain a standard form of integral equation, let us change the variable again. Let

$$
\begin{aligned}
& u=a^{2}-w^{2}, \\
& x=a^{2}-b_{0}^{2} .
\end{aligned}
$$

Changing the variable $w$ to $u, d u=-d w^{2}$; then the above L.H.S. becomes

$$
\pi g(x)=\pi \cdot \int_{0}^{x}(x-u)^{-1 / 2} p(u) d u \quad\left(0 \leq x \leq a^{2}\right) .
$$

The left-hand side of (12) should be a function of $x$ and is denoted by $g(x)$. From (12), we should have

$$
g(0)=0 .
$$

Substituting (12) into the left-hand side of (6), we have

$$
g(x)=(1 / \pi) F(r) \quad\left(0 \leq x \leq a^{2}\right) .
$$

Now, $g(x)$ is a known function by (14), and thus (12) is a standard Abel integral equation and its solution is well known (e.g., in [4]):

$$
p(x)=(2 \pi)\left[\sqrt{x} g^{\prime}(0)+\int_{0}^{x} \sqrt{x-u} g^{\prime \prime}(u) d u\right] \quad\left(0 \leq x \leq a^{2}\right),
$$

where $g^{\prime}=d g / d x, x=a^{2}-b_{0}^{2}=a^{2}-r^{2} \cos ^{2} \theta_{0}$, and $\cos \theta_{0}$ is determined by $g(0)=0$. 
Example. The Hertz contact problem of two elastic balls is considered as an example; i.e., we take

$$
F(r)=\left(\alpha-\beta r^{2}\right) /\left(k_{1}+k_{2}\right),
$$

where $\alpha, \beta, k_{1}$, and $k_{2}$ are constants, and the geometric condition of the problem gives $\alpha=2 \beta a^{2}$, where $a$ is the radius of the contact base.

By (14), we have $g(x)=A x, g^{\prime}(x)=A, g^{\prime \prime}=0$, where

$$
\begin{aligned}
A & =2 \beta /\left[\pi\left(k_{1}+k_{2}\right)\right], \\
x=a^{2}-b_{0}^{2} & =a^{2}-r^{2} / 2 \quad\left(0 \leq x \leq a^{2}\right) .
\end{aligned}
$$

Then by (15), we have

$$
\begin{gathered}
p(x)=(2 A / \pi) \sqrt{x} \quad\left(0 \leq x \leq a^{2}\right), \\
p\left(b_{0}\right)=(2 A / \pi) \sqrt{a^{2}-b_{0}^{2}} \quad\left(0 \leq b_{0} \leq a\right) .
\end{gathered}
$$

Replacing the character $b_{0}$ by $r$, we have

$$
p(r)=\left\{4 \beta /\left[\pi^{2}\left(k_{1}+k_{2}\right)\right]\right\} \sqrt{a^{2}-r^{2}} \quad(0 \leq r \leq a),
$$

which is exactly the same as Hertz's solution [1]. (Note that the expression

$$
p(r)=(2 A / \pi) \sqrt{a^{2}-r^{2} / 2} \quad(0 \leq r \leq \sqrt{2} a),
$$

obtained by substituting $x=a^{2}-r^{2} / 2$ into (19a), does not represent the radical distribution of contact pressure $p(r)$, since the domain of $p(r)$ is $(0 \leq r \leq a)$ and $p(r)$ should satisfy the condition of zero pressure at the contact boundary, i.e., $p(r=a)=0$. Therefore (20) represents the radical distribution of contact pressure.)

\section{SOlution OF SMALl PARAMETERED INTEGRAL EQUATION (5)}

Suppose that

$$
p=p_{0}+\varepsilon p_{1}+\varepsilon^{2} p_{2}+\cdots .
$$

Substituting (22) into (5) and comparing the order of $\varepsilon^{n}$, we have

$$
\begin{gathered}
\iint p_{0} d s d \psi=G(r), \\
\iint p_{n} d s d \psi=-r \iint p_{n-1} d s \cos \psi d \psi \quad(n \geq 1),
\end{gathered}
$$

where the limits of integration are the same as (1) and are omitted and $p_{n}$ $(n \geq 0)$ is subjected to constraints (2) and (3).

By (15), the solution $p_{0}$ of (23) is

$$
p_{0}\left(x_{0}\right)=(2 / \pi)\left[g_{0}^{\prime}(0) \sqrt{x_{0}}+\int_{0}^{x_{0}} \sqrt{x_{0}-u} g_{0}^{\prime \prime} \cdot(u) d u\right] \quad\left(0 \leq x_{0} \leq a^{2}\right)
$$

where $g_{0}\left(x_{0}\right)=(1 / \pi) G(r), x_{0}=a^{2}-r^{2} \cos \theta_{0}$, and $\cos ^{2} \theta_{0}$ is determined by $g_{0}(0)=0$.

Once $p_{n-1} \quad(n \geq 1)$ has been found, then, under certain conditions (the following Existence Theorem), the right-hand side of (24) is integrable and can 
be written as a known function of $r$ so that (24) can be solved in the same way as (23). Repeating the process of solving (24), we can obtain $p_{n}$ for $n \geq 1$.

In the following, we state a theorem, which guarantees the existence of $p_{n}$ for $n \geq 1$.

Existence Theorem. Suppose that the given function $g_{0}\left(x_{0}\right)=(1 / \pi) G(r)$ is continuous and has continuous derivatives $g_{0}^{\prime}, g_{0}^{\prime \prime}$ in $\left[0, a^{2}\right]$. Then

(1) (23) has a continuous solution $p_{0}\left(x_{0}\right)$ in $\left[0, a^{2}\right]$.

(2) (24) has a continuous solution $p_{n}\left(x_{n}\right)$ in $\left[0, a^{2}\right]$ for $n \geq 1$, where $x_{n}=$ $a^{2}-r^{2} \cos ^{2} \theta_{n}, \cos ^{2} \theta_{n}$ is determined by $g_{n}(0)=0, g_{n}\left(x_{n}\right)=(1 / \pi) F_{n}(r)$, and $F_{n}(r)=-r \iint p_{n-1} d s \cos \psi d \psi$.

Using the first integral mean value theorem of functions, changing variables, etc., we can prove this theorem. However, to conserve space, the proof is omitted.

\section{EXAMPLE}

Hertz's contact problem of two elastic balls is again considered as an example for the small parametered integral equation (5).

From (20), instead of $r$ by $t$, we have

$$
p_{0}(t)=(2 A / \pi)\left(a^{2}-t^{2}\right)^{1 / 2} \quad(0 \leq t \leq a) .
$$

Substituting $p_{0}$ into (24) and using (14), we have:

$$
\begin{aligned}
& g_{1}\left(x_{1}\right)= \frac{1}{\pi}\left[-r \int_{0}^{\pi} \int_{-\infty}^{\infty} p_{0}(t) d s \cos \psi d \psi\right] \\
&=-\frac{4 A}{\pi^{2}} \int_{0}^{\pi} \int_{0}^{V}\left(a^{2}-r^{2} \sin ^{2} \psi-v^{2}\right)^{1 / 2} d v \cos \psi d \psi \\
&= K \cdot x_{1}\left(a^{2}-x_{1}\right)^{1 / 2} \\
& g_{1}^{\prime}\left(x_{1}\right)=K\left(a^{2}-x_{1}\right)^{1 / 2}-\left(x_{1} / 2\right)\left(a^{2}-x_{1}\right)^{-1 / 2} \\
& g_{1}^{\prime}(0)=K a \\
& g_{1}^{\prime \prime}\left(x_{1}\right)=K\left(a^{2}-x_{1}\right)^{-1 / 2}+\left(x_{1} / 4\right)\left(a^{2}-x_{1}\right)^{-3 / 2}
\end{aligned}
$$

where

$$
\begin{gathered}
K=-\left(4 \sqrt{3} A / \pi^{2}\right)(1+\pi / 2), \\
x_{1}=a^{2}-r^{2} / 3,
\end{gathered}
$$

$t^{2}=v^{2}+r^{2} \sin ^{2} \psi, v=s+r \cdot \cos \psi$ (Figure 1), and $x_{1}=a^{2}-r^{2} \cos ^{2} \theta_{1}$ $\left(\cos ^{2} \theta_{1}=1 / 3\right.$ is determined by $\left.g_{1}(0)=0\right)$ have been used.

Substituting (26)-(28) into (15), we have

$$
\begin{aligned}
p_{1}\left(x_{1}\right)=\frac{2 K}{\pi}\left\{\left(a^{2}-x\right) \log \frac{\sqrt{a^{2}-x_{1}}}{a-\sqrt{x_{1}}}\right. \\
\left.+\frac{3}{4}\left[\left(\frac{5 x_{1}}{6}-\frac{a^{2}}{2}\right) \cdot \log \frac{-\sqrt{x_{1}}-a}{\sqrt{x_{1}}+a}+a \sqrt{x_{1}}\right]\right\}
\end{aligned}
$$

$\left(0 \leq x_{1} \leq a^{2}\right)$, and so on. 
Finally, the solution of (5) can be obtained by (22).

Let us make a comparison on maximum pressure $p(0)$ at the centre $r=0$, i.e., $x=a^{2}$, of the contact range, between Hertz's solution $p_{0}(0)$ and the asymptotic solution $p(0)=p_{0}(0)+\varepsilon p_{1}(0)$ of this paper:

$$
p_{0}(0)=(2 A / \pi) a, \quad p_{1}(0)= \pm(3 K /(2 \pi)) a^{2} .
$$

If the relative error $e$ is defined by

$$
e=\left|\left[p(0)-p_{0}(0)\right] / p_{0}(0)\right|=\left|\varepsilon p_{1}(0) / p_{0}(0)\right|
$$

and the expression of small parameter $\varepsilon$ is listed by [2]

$$
\begin{aligned}
\varepsilon= & \frac{1}{2}\left[\frac{\left(1-2 \nu_{1}\right)\left(1+\nu_{1}\right) E_{2}}{R_{1}}+\frac{\left(1-2 \nu_{2}\right)\left(1+\nu_{2}\right) E_{1}}{R_{2}}\right] \\
& \cdot\left\{\left[\left(E_{1}\left(1-\nu_{1}^{2}\right)\right]^{-1}+\left[E_{2}\left(1-\nu_{2}^{2}\right)\right]^{-1}\right\},\right.
\end{aligned}
$$

where $E_{1}, E_{2}, \nu_{1}, \nu_{2}$, and $R_{1}, R_{2}$ are the elastic modulus, Poisson's ratio, and the radius of the two elastic balls respectively, then substituting $p_{0}(0)$, $p_{1}(0)$, and $\varepsilon$ into (32), for $E_{1}=E_{2}, \nu_{1}=\nu_{2}=0.3, R_{1}=R_{2}=10 a$, we have $e=7.7342 \%$. This result shows that from the criterion of the more exact integral equation (5), the classical Hertz solution is not exact enough for engineering applications and the asymptotic solution is better.

\section{REFERENCES}

1. S. P. Timoshenko and J. N. Goodier, Theory of elasticity, 3rd ed., McGraw-Hill, New York, 1970, pp. 411-412.

2. T. Q. Yun, The exact integral equation of Hertz's contact problem, Appl. Math. Mech. 12 (1991), 181-185.

3. G. T. Herman, Image reconstruction from projections, the fundamentals of computerized tomography, Academic Press, New York, 1980.

4. T. Q. Yun, Integral equations and their applications in mechanics, South China University of Technology Publishers, Guangzhou, 1990, p. 60. (Chinese)

Department of Mechanics, South China University of Technology, Guangzhou, 510641, The People's Republic of China 